\title{
Ground State of the Strong-Coupling Hubbard Hamiltonian: A Numerical Diagonalization Study
}

\section{Citation}

Kaxiras, Efthimios and Efstratios Menousakis. Ground state of the strong-coupling Hubbard Hamiltonian: A numerical diagonalization study. Physical Review B 37(1): 656-659.

\section{Published Version}

http://link.aps.org/doi/10.1103/PhysRevB.37.656

\section{Permanent link}

http://nrs.harvard.edu/urn-3:HUL.InstRepos:2943923

\section{Terms of Use}

This article was downloaded from Harvard University's DASH repository, and is made available under the terms and conditions applicable to Other Posted Material, as set forth at http:// nrs.harvard.edu/urn-3:HUL.InstRepos:dash.current.terms-of-use\#LAA

\section{Share Your Story}

The Harvard community has made this article openly available.

Please share how this access benefits you. Submit a story.

\section{Accessibility}




\title{
Ground state of the strong-coupling Hubbard Hamiltonian: A numerical diagonalization study
}

\author{
Efthimios Kaxiras \\ IBM Research Division, Thomas J. Watson Research Center, Yorktown Heights, New York 10598 \\ Efstratios Manousakis \\ Supercomputer Computations Research Institute, Florida State University, Tallahassee, Florida 32306
}

(Received 6 October 1987)

\begin{abstract}
We exactly diagonalize the effective Hamiltonian obtained from the Hubbard model in the strong-coupling limit for a two-dimensional $\sqrt{10} \times \sqrt{10}$ size square lattice. The effective Hamiltonian operates on a restricted Hilbert space containing only states with singly occupied sites, which makes the diagonalization possible within reasonable computational time for the above lattice. The ground-state energy and wave function are obtained for several values of the coupling ratio $t / U$ and the doping fraction $x$. We find three different phases in the $(x, t / U)$ phase diagram. The first is characterized by antiferromagnetic order which extends to the longest possible distance in the 10-site system. The second and third phases are characterized by antiferromagnetic and ferromagnetic short-range correlations, respectively. We comment on the possible relevance of the results to the recently discovered high-temperature copper-oxide superconductors.
\end{abstract}

The recent discovery of high-temperature superconductivity ${ }^{1}$ in copper oxides has kindled new interest in the numerical solution of simple theoretical models. There is a growing suspicion that a phonon mechanism alone cannot account for such high critical temperatures. The possibility of new pair-binding mechanisms of electronic origin is the object of intense theoretical investigations. A common point of departure for many theoretical studies is the Hubbard model which had been proposed to describe electron correlations in narrow-band systems. This simple model has been extensively studied over the past twenty years mostly in connection with the metal-insulator transition. It is argued ${ }^{2}$ that the same simple model could possibly capture the physics of high-temperature superconducting materials.

Beyond one spatial dimension, however, there is no exact solution to the Hubbard Hamiltonian, and information from analytical studies in the various approximations or limits of the model is sometimes conflicting. Exact numerical diagonalizations are available only for mediumsized systems in the limit of infinite on-site repulsion, ${ }^{3}$ or for very small systems, ${ }^{4}$ because the dimensionality of the Hilbert space grows exponentially with the size of the system. Monte Carlo (MC) attempts ${ }^{5}$ to simulate the model at finite temperatures are hindered by well-known problems due to fermion statistics, and the calculations are restricted to two dimensions and small systems. The structure of the ground state cannot be easily revealed in these two-dimensional MC studies. Large statistical errors are involved in taking the zero-temperature limit due to finite-size effects in the "Trotter" (temperature) direction of the lattice. Variational Monte Carlo studies ${ }^{6,7}$ of medium-sized systems have been reported recently and provide interesting insight to the problem.

In the superconducting copper oxides the ratio of the hopping matrix element $t$ to the on-site Coulomb repulsion energy $U$ is thought to be small. ${ }^{2}$ Accordingly, a reduced Hamiltonian derived from the Hubbard model has been analytically studied by several authors. ${ }^{8,9}$ This Hamiltonian is correct to leading order in the $t / U$ expansion and acts on a subspace of the Hilbert space in which there are only singly occupied sites. The reduced Hamiltonian consists of a hopping term and two-site and threesite electron exchange terms. ${ }^{9}$ At half-filling it is formally identical to the antiferromagnetic Heisenberg model (AHM) which, for a square or rectangular lattice, is expected to have antiferromagnetic long-range order in the ground state. Departure from half-filling by light or heavy doping introduces the possibility of a transition to a superconducting state as suggested in Refs. 8 and 9. In these analytical studies assumptions concerning the structure of the ground state enter in a crucial way. On the other hand, the effective Hamiltonian seems to give rise to a wealth of interesting physics, which might be masked by symmetries that one has built into the approximate ground state. It is instructive therefore to resort to numerical studies.

In the present work, we investigate the properties of the reduced Hubbard Hamiltonian on a $\sqrt{10} \times \sqrt{10}$ square lattice with periodic boundary conditions by exactly diagonalizing the reduced Hamiltonian matrix. The fact that this Hamiltonian acts on a restricted Hilbert space makes the diagonalization possible. We obtain the ground-state energy and wave function as a function of the parameter $t / U$ and at various fillings of the lattice. There are two interesting aspects of the ground state: its magnetic nature and the possible existence of quasiparticles such as electron pairs or boson excitations whose existence is linked to superconductivity. At present, neither aspect is unequivocally resolved. In this work we focus on the first issue: namely, the magnetic properties of the ground state. We find that the antiferromagnetic order of the half-filled state persists at small to moderate doping, up to the longest possible distance in the 10-site system. This is seen for the first time in an exact solution of the reduced Hubbard Hamiltonian, and will be an interesting 
result if it survives in the limit of an infinite system.

We start from the Hubbard model on a two-dimensional (2D) square lattice, with $M$ sites and $N$ electrons. The Hamiltonian of the system is given by

$$
H=U \sum_{i} N_{i \uparrow} N_{i \downarrow}-t \sum_{\langle i j\rangle, s}\left(C_{i, s}^{\dagger} C_{j, s}+\text { H.c. }\right),
$$

where $C_{i, s}$ is the annihilation operator for an electron at site $i$ with spin $s, N_{i, s}$ the corresponding occupation number operator, and $\langle i j\rangle$ denotes a pair of nearest-neighbor sites. The first term in Eq. (1) discourages double occupancy of any site, and the second term allows the electrons to hop from site to site. The high- $T_{c}$ oxide superconductors have strong two-dimensional features, so that in applying the present results to these materials, $t$ should be thought of as the hopping matrix element between copper atoms in a $\mathrm{Cu}-\mathrm{O}$ plane. Implementation of additional features of these materials may be necessary to provide full understanding of the superconducting mechanism.

In the limit $t / U \ll 1$ the second term in Eq. (1) is small and can be treated by standard perturbation theory. The unperturbed Hamiltonian has highly degenerate energy levels equally spaced by $U$. The lowest level corresponds to states with no doubly occupied sites, the first level corresponds to states with exactly one doubly occupied site, etc. Let $S_{0}$ be the Hilbert subspace spanned by the states of lowest energy. To second order, the linear combination among the states of $S_{0}$ that best approximates the ground state is obtained by diagonalizing the following effective Hamiltonian: ${ }^{9}$

$$
\begin{aligned}
& H_{\mathrm{eff}}=H_{1}+H_{2}+H_{3}, \\
& H_{1}=-t \sum_{\langle i j\rangle, s}\left[\xi_{i} C_{i, s}^{\dagger} C_{j, s}+(i \leftrightarrow j)\right], \\
& H_{2}=-2 \frac{t^{2}}{U} \sum_{\langle i j\rangle, s}\left(C_{j, s}^{\dagger} C_{i, s} N_{i,-s} C_{i, s}^{\dagger} C_{j, s}\right. \\
& \left.\quad+C_{j,-s}^{\dagger} C_{i,-s} C_{i, s}^{\dagger} C_{j, s}\right), \\
& H_{3}=-\frac{t^{2}}{U} \sum_{\langle i j k\rangle, s}\left[\xi _ { k } \left(C_{k, s}^{\dagger} C_{j, s} N_{j,-s} C_{j, s}^{\dagger} C_{i, s}\right.\right. \\
& \left.\left.\quad+C_{k,-s}^{\dagger} C_{j,-s} C_{j, s}^{\dagger} C_{i, s}\right)+(i \leftrightarrow k)\right],
\end{aligned}
$$

where $\langle i j k\rangle$ denotes a triplet of sites in which $i j$ and $j k$ are nearest neighbors, and $\xi_{i}=1$ or 0 depending on whether the site is empty or occupied. The first term allows the electron to move to an empty nearest-neighbor (NN) site only. Both $\mathrm{H}_{2}$ and $\mathrm{H}_{3}$ describe processes in which double occupancy occurs only virtually, resulting in an energy cost $U$. The two-site term $\mathrm{H}_{2}$ describes processes in which an electron hops to a neighboring site occupied by an electron of opposite spin, making the site momentarily doubly occupied. In the final state the two electrons are either in the original configuration or in the one with spins exchanged. $\mathrm{H}_{3}$ gives rise to three-site virtual processes in which one electron hops to a NN site occupied by an opposite spin electron, and the resulting double occupation is relieved with the hopping of either electron to a next NN empty site. More details about this Hamiltonian are given in Refs. 8 and 9.

At half-filling, $\mathrm{H}_{1}$ and $\mathrm{H}_{3}$ are zero and the remaining piece $\mathrm{H}_{2}$ has the same matrix elements in $S_{0}$ as the Hamiltonian of the AHM with coupling $J=4 t^{2} / U$. In the infinite- $U$ limit, on the other hand, and at a nonzero doping fraction $x=1-N / M$, only the first term remains. This is still a nontrivial problem because doubly occupancy is not allowed. Both these problems have been solved by numerical diagonalization: Oitmaa and Betts ${ }^{10} \mathrm{calcu}$ lated the ground-state properties of the AHM on a $4 \times 4$ square lattice and Takahashi ${ }^{3}$ solved the $U=\infty$ limit for arbitrary filling on finite cubic lattices of up to 12 sites.

In this work, we diagonalize the Hamiltonian (2)-(5) for arbitrary $U$ and $x$. Of course, one has to keep in mind that its validity is restricted to small values of the ratio $t / U$. Nevertheless, the numerical solution will be helpful because the Hamiltonian itself is insoluble, and, in order to reach useful conclusions, further limits or approximations are needed.

Let us briefly discuss the choice of the lattice. Consider the infinite two-dimensional square lattice in which the solid square shown in Fig. 1 will be identified as the unit cell. This unit cell has dimensions $\sqrt{10} \times \sqrt{10}$, contains 10 sites, and can tile the infinite two-dimensional square lattice $^{10}$ [the squares which can tile the infinite 2D square lattice have linear dimension in lattice units $\left(n^{2}+m^{2}\right)^{1 / 2}$ where $n$ and $m$ are integers]. The calculations were performed on a single unit cell imposing periodic boundary conditions. Ten is at present the largest number of sites we can easily study for all possible fillings and couplings. This unit cell was preferred over other choices having different geometries but the same number of sites (e.g., $2 \times 5$ ) in order to avoid having to introduce anisotropy among the coupling constants for the two directions. Also, it is important to restrict the choice of unit cells to

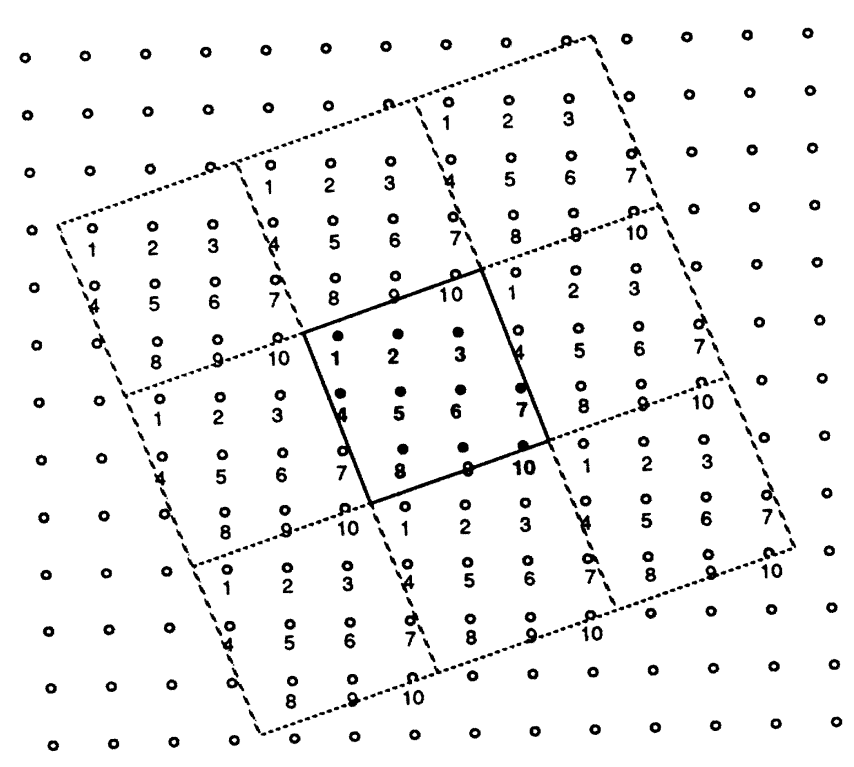

FIG. 1. The two-dimensional unit cell. The infinite 2D lattice can be tiled by these tilted squares of size $\sqrt{10} \times \sqrt{10}$ in lattice units. 
those containing an even numbers of sites, because only then are the two sublattices of the infinite square lattice clearly distinguishable. This feature is essential for the calculation of the spin-spin correlations and for avoiding frustration of the possible antiferromagnetic order at the boundaries.

In order to facilitate the computations, we have used the Lanczos scheme for matrix diagonalization, as implemented in Ref. 11. This proved very efficient since the number of nonzero matrix elements was less than $1 \%$ of the total for the largest matrices considered, which were $4200 \times 4200$. We found that the simplest way to calculate the Hamiltonian matrix was to consider subspaces of eigenstates all having the same $S_{z}$. This method does not take full advantage of the symmetries of the problem; further reduction of matrix size can be achieved by considering invariant subspaces characterized by operators which commute with the Hamiltonian. The latter, however, results in a more complicated procedure for the evaluation of the matrix elements and becomes advantageous only for larger lattices.

For each filling factor $n_{e}=N / M=1-x$, we have considered the Hamiltonian for different values of $t / U$ ranging from 0 to $\sim 0.5$, above which the validity of the reduced Hamiltonian (2)-(5) becomes questionable, since $t$ and $U$ are then of the same order. As a check of our calculations, we have exactly reproduced the results of Oitmaa and Betts ${ }^{10}$ for the 8-site and 10-site lattices at halffilling $\left(n_{e}=1\right.$, one electron per site), and those of Takahashi ${ }^{3}$ for the $U \rightarrow \infty$ case, at all possible filling factors.

Our results show that the lowest-energy state obtained by diagonalizing the Hamiltonian is always characterized by the smallest possible value of $\left|S_{z}\right|$. In the case of half-filling, it has been shown rigorously that the ground state has total $S=0 .{ }^{12}$ Detailed information about magnetic properties can best be obtained by examining the wave function of the ground state and in particular spinspin correlation functions. Let the ground state be

$$
\left|\psi_{0}\right\rangle=\sum_{\left\{n_{i, s}\right\}} C\left(\left\{n_{i, s}\right\}\right)\left|\left\{n_{i, s}\right\}\right\rangle,
$$

where $n_{i, s}=0,1$ are the eigenvalues of the number operator $N_{i, s}$. We then define the spin-spin correlation function $G_{s}(r)$ :

$$
G_{s}(r)=\left\langle\psi_{0}\left|s_{z}(0) s_{z}(r)\right| \psi_{0}\right\rangle
$$

where $s_{z}(i)$ is the $z$ component of the spin operator at site $i$.

The spin-spin correlation function shows the following interesting features (see also Table I): At half-filling $(x=0$, first line of Table I), there is tendency for antiferromagnetic ordering, the first NN's being antiparallel $\left[G_{s}(1)<0\right.$ ] and the second and third NN's being parallel $\left[G_{s}(\sqrt{2})-G_{s}(2)>0\right]$. Notice that the structure of the lattice and the periodic boundary conditions give $G_{s}(\sqrt{2}) \equiv G_{s}(2)$. For nonzero values of the doping fraction $x$ (i.e., a certain number of holes) the antiferromagnetic ordering similar to that at half-filling extends up to some value of $x$, depending on $t / U$ (second line of Table I, AF phase). As $x$ or $t / U$ is varied, the system can cross to
TABLE I. The correlation function for the first, second, and third NN at different phases. The dash in the first entry represents any value of $t / U$.

\begin{tabular}{lcccc}
\hline \hline & $(x, t / U)$ & $G_{s}(1)$ & $G_{s}(\sqrt{2})=G_{s}(2)$ & Phase \\
\hline 1 & $(0,-)$ & -0.487 & +0.313 & AF \\
2 & $(0.1,0.10)$ & -0.301 & +0.167 & AF \\
3 & $(0.2,0.075)$ & -0.118 & -0.038 & AFC \\
4 & $(0.2,0.015)$ & 0.037 & -0.097 & FC \\
\hline \hline
\end{tabular}

a phase where the first-neighbor antiferromagnetic correlation persists [AFC phase, $G_{s}(1)<0$ ] but furtherneighbor correlations nearly vanish $\left[G_{s}(\sqrt{2})-G_{s}(2) \leq 0\right.$, see third line of Table I]. Finally, there is a third possible phase in which the short-range order changes from antiferromagnetic to ferromagnetic [FC phase, $G_{s}(1)>0$ ]. The longer-range order in the last phase indicates the existence of pairs of parallel spins each surrounded by opposite pairs $\left[G_{s}(\sqrt{2})=G_{s}(2) \leq 0\right.$, see fourth line of Table I]. The full phase diagram is shown in Fig. 2 and representative states well inside each phase are indicated by dots numbered according to the tabulation in Table $I$. The phase boundaries are defined by changes in the sign of the first-neighbor correlation (lower boundary between AFC and FC) and the sign of the second- and third-neighbor correlation (upper boundary between AF and AFC). The lower boundary is also characterized by an abrupt change in the magnitude as well as the sign of the correlation function.

To make the picture clearer, we calculate the probability distribution of the staggered magnetization in the ground state. The staggered magnetization operator $\hat{M}_{\text {st }}$ per site is defined as

$$
\hat{M}_{\mathrm{st}}=\frac{1}{M}\left(\sum_{i \in A} s_{z}(i)-\sum_{i \in B} s_{z}(i)\right),
$$

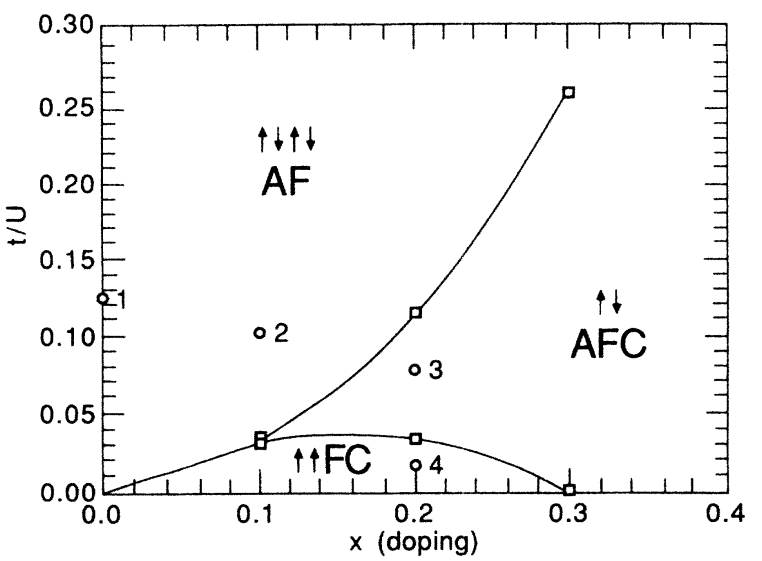

FIG. 2. The phase diagram on the $(x, t / U)$ plane. AF stands for antiferromagnetic phase, AFC and FC for the phases with antiferromagnetic and ferromagnetic short-range correlations. The points indicated by squares are transition points determined by changes in the correlation functions (see text). The numbered dots correspond to representative states inside each phase (see Table I). The lines are guide to the eye. 
where $A$ and $B$ denote the two sublattices. The proper quantity to discern the presence of spontaneous staggered magnetization in a finite system is the staggered magnetization distribution

$$
P\left(S_{m}\right) \equiv \sum_{\left\{n_{i, s}\right\}}\left|C\left(\left\{n_{i, s}\right\}\right)\right|^{2} \delta_{S_{m}, M_{u}\left(\left\{n_{i, s}\right\}\right),}
$$

where $M_{\mathrm{st}}\left(\left\{n_{i, s}\right\}\right)$ is the staggered-magnetization eigenvalue of the state $\left|\left\{n_{i, s}\right\}\right\rangle$. In other words, the sum is over all configurations having the staggered-magnetization eigenvalue equal to $S_{m}$. In Fig. 3 we plot the calculated staggered-magnetization distribution for $t / U=0.15$ and for $x=0.1,0.2$, and 0.3 . For $x=0.3$ the system is in a phase with no long-range antiferromagnetic order and the staggered magnetization is distributed around $S_{m}=0$. For $x=0.1$ the system is in the AF phase, and the distribution exhibits two peaks at $S_{m}= \pm 0.25$. (In the thermodynamic limit, the up-down symmetry would be spontaneously broken and the two values would be separated by an infinite-energy barrier. Only one of the two possible states will be observed depending upon the initial conditions.) For $x=0.2$ the system is just about to develop staggered magnetization.

A phase diagram remarkably similar to Fig. 2 has been suggested by the variational calculation of Ref. 7. Our phase diagram is also consistent with mean-field theory, ${ }^{13}$ even though our calculations are valid for small $t / U$ and mean-field theory is valid for large $t / U$. In Ref. 5, a MC study of the full Hubbard model at finite temperature finds antiferromagnetism only for $x=0$. The difference might indicate that the extrapolation procedure to the zero-temperature limit in the MC calculation is strongly affected by finite-size effects.

Finally, we consider the possible relevance of the present results for high-temperature superconductivity as can be interpreted in the theoretical framework of Ref. 9. If the ground state of the system liew in the AF phase, the vacancies created by light doping will undergo a BoseEinstein condensation and superconductivity is due to the superfluidity of the charged condensate. If, on the other hand, the ground state of the system lies in the phase with

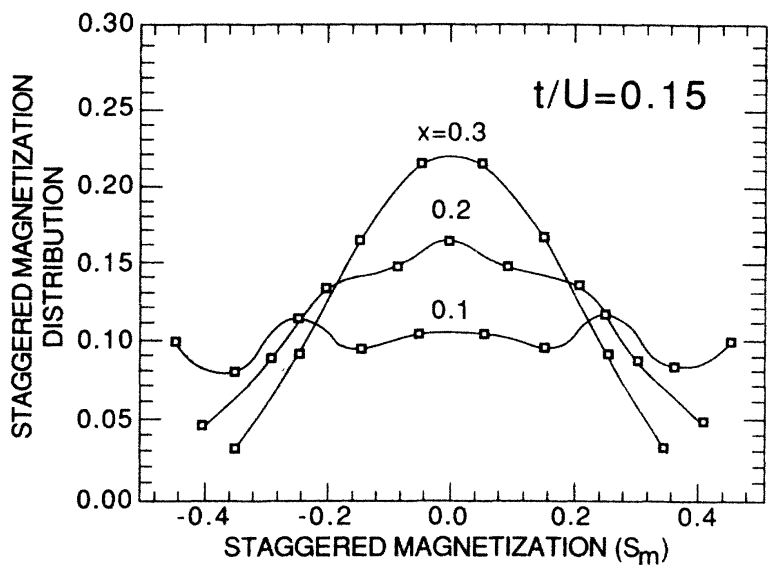

FIG. 3. The calculated staggered magnetization distribution for $t / U=0.15$ and for $x=0.1,0.2$, and 0.3 . The lines are guides to the eye.

short-range AF ( $F$ ) correlations, it is possible that formation of pairs in a singlet (triplet) state will occur. ${ }^{8,14}$ The singlet or triplet pairs can play the role of Cooper pairs in the superconducting state. A realistic study of correlation functions related to condensation of holes or electron pairs may require larger lattices than the one considered in the present work.

We would like to thank Dr. D. H. Lee, Dr. T. D. Schultz, and Professor Y.-L. Wang for a critical reading of the manuscript. We are especially grateful to Dr. D. H. Lee for informing us about unpublished work by $H$. $Q$. Lin, J. E. Hirsch, and D. J. Scalapino, which addresses the question of pair susceptibilities by diagonalizing the full Hubbard hamiltonian on an eight-site lattice. This work was supported in part by the Florida State University Supercomputer Computations Research Institute which is partially funded by the U.S. Department of Energy through Contract No. DE-FC05-85ER250000.
1J. G. Bednorz and K. A. Müller, Z. Phys. B 64, 188 (1986); S. Uchida, H. Takagi, K. Katazawa, and S. Tanaka, Jpn. J. Appl. Phys., Part 2, 26, L1 (1987); Z. Zhao et al., Kexue Tongbao (China) (to be published); C. W. Chu, P. H. Hor, R. L. Meng, L. Gao, Z. J. Huang, and Y. Q. Wang, Phys. Rev. Lett. 58, 405 (1987); R. Cava, R. B. van Dover, B. Batlogg, and E. A. Rietman, ibid. 58, 408 (1987).

2P. W. Anderson, Science 235, 1196 (1987).

${ }^{3}$ M. Takahashi, J. Phys. Soc. Jpn. 51, 3475 (1982).

${ }^{4}$ A. Kawabata, Solid State Commun. 32, 893 (1979).

5 J. E. Hirsch, Phys. Rev. B 31, 4403 (1985).

${ }^{6}$ C. Gross, R. Joint, and T. M. Rice, Phys. Rev. B 36, 381 (1987).

${ }^{7}$ H. Yokoyama and H. Shiba, J. Phys. Soc. Jpn. 56, 3570 (1987).
${ }^{8}$ J. E. Hirsch, Phys. Rev. Lett. 54, 1317 (1985); P. W. Anderson, G. Baskaran, Z. Zou, and T. Hsu, ibid. 58, 2790 (1987); S. Kivelson, D. S. Rokhsar, and J. P. Sethna, Phys. Rev. B 35, 8865 (1987); A. E. Ruckenstein, P. J. Hirschfeld, and J. Appel, ibid. 36, 857 (1987).

${ }^{9}$ K. Huang and E. Manousakis, Phys. Rev. B (to be published).

${ }^{10}$ J. Oitmaa and D. D. Betts, Can. J. Phys. 56, 897 (1978).

${ }^{1}$ See J. Cullum and R. A. Willoughby, Lanczos Algorithms for Large Symmetric Eigenvalue Computations (Birkhauser, Basel, 1985), Vols. 1 and 2.

${ }^{12}$ W. Marshall, Proc. R. Soc. London, Ser. A 232, 48 (1955).

${ }^{13}$ D. Penn, Phys. Rev. 142, 350 (1966).

${ }^{14}$ K. S. Bedell and D. Pines (unpublished). 